\title{
Structural and magnetic properties of $\mathrm{FeMn}_{x}$ chains $(x=1-6)$ supported on $\mathrm{Cu} 2 \mathrm{~N} / \mathrm{Cu}(100)$
}

\author{
Deung-Jang Choi, ${ }^{1}$ Roberto Robles, ${ }^{2}$ Jean-Pierre Gauyacq, ${ }^{3}$ Markus Ternes, ${ }^{4}$ Sebastian Loth, ${ }^{4,5}$ and Nicolás Lorente ${ }^{6,7}$ \\ ${ }^{1}$ CIC nano GUNE, Tolosa Hiribidea 78, 20018 Donostia-San Sebastian, Spain \\ ${ }^{2}$ Catalan Institute of Nanoscience and Nanotechnology (ICN2), CSIC and The Barcelona Institute of Science and Technology, \\ Campus UAB, Bellaterra, 08193 Barcelona, Spain \\ ${ }^{3}$ Institut des Sciences Moléculaires d'Orsay, ISMO, CNRS, Université Paris-Sud, Université Paris-Saclay, \\ Bâtiment 351, 91405 Orsay Cedex, France \\ ${ }^{4}$ Max Planck Institute for Solid State Research, 70569 Stuttgart, Germany \\ ${ }^{5}$ Max Planck Institute for the Structure and Dynamics of Matter, 22761 Hamburg, Germany \\ ${ }^{6}$ Centro de Fisica de Materiales, CFM/MPC (CSIC-UPV/EHU), Paseo Manuel de Lardizabal 5, \\ 20018 Donostia-San Sebastian, Spain \\ ${ }^{7}$ Donostia International Physics Center (DIPC), Paseo Manuel de Lardizabal 4, 20018 Donostia-San Sebastian, Spain
}

(Received 18 May 2016; published 5 August 2016)

\begin{abstract}
Heterogeneous atomic magnetic chains are built by atom manipulation on $\mathrm{Cu}_{2} \mathrm{~N} / \mathrm{Cu}(100)$ substrate. Their magnetic properties are studied and rationalized by a combined scanning tunneling microscopy (STM) and density functional theory (DFT) work completed by model Hamiltonian studies. The chains are built using Fe and $\mathrm{Mn}$ atoms ontop of the $\mathrm{Cu}$ atoms along the $\mathrm{N}$ rows of the $\mathrm{Cu}_{2} \mathrm{~N}$ surface. Here, we present results for FeMn chains $(x=1-6)$ emphasizing the evolution of the geometrical, electronic, and magnetic properties with chain size. By fitting our results to a Heisenberg Hamiltonian we have studied the exchange-coupling matrix elements $J$ for different chains. For the shorter chains, $x \leqslant 2$, we have included spin-orbit effects in the DFT calculations, extracting the magnetic anisotropy energy. Our results are also fitted to a simple anisotropic spin Hamiltonian and we have extracted values for the longitudinal-anisotropy $D$ and transversal-anisotropy $E$ constants. These parameters together with the values for $J$ allow us to compute the magnetic excitation energies of the system and to compare them with the experimental data.
\end{abstract}

DOI: 10.1103/PhysRevB.94.085406

\section{INTRODUCTION}

Bottom-up approaches give access to systems of very reduced dimensionality with unique physical properties. Among these systems, chains of a single-atom cross section are of great interest [1]. When the chains are formed by magnetic atoms, spin-spin correlations can come into place leading to new phenomena and applications [2]. Building structures from their atomic constituents can be achieved by the atomic-manipulation capabilities of the scanning tunneling microscope (STM). Magnetic atoms have been positioned one by one at different distances and with different arrangements on a variety of substrates [3-7]. Spin-polarized STM [8] or inelastic electron tunneling spectra (IETS) $[9,10]$ have granted us a detailed vision of the magnetic mechanisms at play on the atomic scale.

Recent developments in density-functional theory (DFT) have also permitted us to attain a deep understanding of the phenomena revealed by the above experiments. Due to their experimental interest, Mn chains are amongst the most studied ones [11-16]. Fe chains are also well known both experimentally and theoretically [4,5,15,17-21]. The work by Lin and Jones [13,22] on $\mathrm{Fe}, \mathrm{Co}$, and $\mathrm{Mn}$ atoms on $\mathrm{Cu}_{2} \mathrm{~N} / \mathrm{Cu}$ (100) reveals that atomic spins maintain their nominal values on the surface $\left(S_{\mathrm{Fe}}=2, S_{\mathrm{Mn}}=5 / 2\right.$, and $S_{\mathrm{Co}}=$ $3 / 2$ ), showing the interest of using $\mathrm{Cu}_{2} \mathrm{~N}$ to preserve much of the magnetic identity of transition-metal (TM) atoms. Studies of Fe chains [17] and of Mn [12] reveals that close-packed TM chains on $\mathrm{Cu}_{2} \mathrm{~N} / \mathrm{Cu}$ (100) couple antiferromagnetically due to a $\mathrm{N}$-mediated superexchange mechanism, largely explaining experimental findings [5,22].
In the present work, we report on a different type of magnetic atomic chain. These chains are heterogeneous, including two types of magnetic atoms, $\mathrm{Fe}$ and $\mathrm{Mn}$, on a $\mathrm{Cu}_{2} \mathrm{~N} / \mathrm{Cu}$ (100) substrate. An initial account of the results has been given in a separate publication [23]. The experimental study is based on STM and IETS results of $\operatorname{FeMn}_{x}(x=1-6)$ and confirms that, as in the previous cases, the magnetic ordering along the chain is antiferromagnetic. The different anisotropy of $\mathrm{Fe}$ and $\mathrm{Mn}$ on $\mathrm{Cu}_{2} \mathrm{~N} / \mathrm{Cu}$ (100) leads to two possible orientations of the magnetic moments (along the chain and out of plane). Contrary to the case of homogeneous chains, this may indicate a noncollinear arrangement. When $x$ is an odd number, a simple-minded evaluation of the total spin yields $1 / 2$, which is compatible with the appearance of a Kondo feature at zero bias [23]. However, when $x$ is even, the expected spin is 2 and, correspondingly, no Kondo peak is observed. In the present work, we perform extensive calculations and compare them with the experiments. The comparison permits us to conclude on the spin arrangement (it is collinear along the chain) and on the behavior of the exchange coupling between the two different atomic species of the chain in good agreement with the experimental observations. The antiferromagnetic coupling is confirmed and traced back to the N-mediated superexchange mechanism as in the homogeneous chains. Finally, the obtention of model Hamiltonians to study the magnetic structure of these chains is discussed within the framework of DFT calculations [18,24].

\section{EXPERIMENTAL METHOD}

Experiments were performed in an ultrahigh vacuum lowtemperature STM at a base temperature of $0.5 \mathrm{~K}$ as has been 
partially reported in Ref. [23]. Differential conductance was used as a local spectroscopic tool that gives information via inelastic electron tunneling (IETS) $[9,10]$. The differential conductance was directly measured using lock-in detection with $72-\mu \mathrm{V}$ rms modulation at $691 \mathrm{~Hz}$ to the sample bias voltage $V$.

The $\mathrm{Cu}$ (100) surface was cleaned by Ar sputtering and then annealed up to $850 \mathrm{~K}$. After having big terraces of $\mathrm{Cu}(100)$ crystal, a monolayer of $\mathrm{Cu}_{2} \mathrm{~N}$ was formed as a decoupling layer by nitrogen sputtering and post-annealing at $600 \mathrm{~K}$. Single Fe and $\mathrm{Mn}$ atoms were deposited onto the pre-cooled surface. Pt-Ir tips were prepared by sputter-anneal cycles and coated with copper in vacuo by soft indentations into the $\mathrm{Cu}$ bridges. The tip status was monitored through STM images and controlled to manipulate the atoms. All atomic chains were built using vertical atomic manipulation. After identifying a given adatom by its spectroscopic features, we picked it up by voltage pulsing, dropped it off on a nitrogen site and hop it to a copper site. We build the chain atom by atom in a close-packed configuration to ensure an AF coupled spin chain. By doing so, all different kinds of $\mathrm{FeMn}_{x}(x=1-10)$ chains were constructed.

\section{THEORETICAL METHOD}

$A b$ initio calculations are performed within the densityfunctional theory (DFT) framework as implemented in the VASP code [26]. We expand the wave functions using a plane-wave basis set with a cutoff energy of $300 \mathrm{eV}$. Core electrons are treated within the projector augmented wave method [27,28]. The PBE form of the generalized gradient approximation is used for the exchange and correlation functional [29].

The system consists of a $\mathrm{Cu}(100)$ surface covered by a $\mathrm{Cu}_{2} \mathrm{~N}$ layer. To model the surface, we use a slab geometry with four $\mathrm{Cu}$ layers plus the $\mathrm{Cu}_{2} \mathrm{~N}$ layer. Following the above experimental procedure, $\mathrm{Fe}$ and $\mathrm{Mn}$ atoms are deposited on top $\mathrm{Cu}$ atoms, forming a chain in the [010] direction. We use a unit cell $[3 \times(x+3)]$ in units of the bulk lattice parameter such that the length along the [010] direction increases as $(x+3)$, where $x$ is the number of $\mathrm{Mn}$ atoms. In this way, we keep the distance between chain images constant for all sizes, being of three lattice constants in the unrelaxed configuration. The unit cell for FeMn 2 is shown in Fig. 2 as an example. The bottom $\mathrm{Cu}$ layer was kept fixed and the remaining atoms were allowed to relax until forces were smaller than $0.01 \mathrm{eV} / \AA$. The $k$-point sample was varied accordingly to the unit cell, and tests were performed to assure its convergence. The charge and magnetic moments have been calculated using the Bader analysis [30].

A critical aspect of the calculations is the use of a static Coulomb charging energy $U$. Lin and Jones [13] perform a constrained DFT calculation to evaluate $U$. For Mn, they find $U=4.9 \mathrm{eV}$ when $\mathrm{Mn}$ is sitting ontop a surface $\mathrm{Cu}$ atom, while it is reduced to $U=3.9 \mathrm{eV}$ ontop a $\mathrm{N}$ site. This is in agreement with their previous result [22], where they found $U=5.0 \mathrm{eV}$ for $\mathrm{Mn}$ ontop of $\mathrm{Cu}$, and $U=2.0 \mathrm{eV}$ for $\mathrm{Fe}$. To the best of our knowledge, these are the only actual computations of $U$ for $\mathrm{Mn}$ on the $\mathrm{Cu}_{2} \mathrm{~N} / \mathrm{Cu}$ (100) substrate. Rudenko et al. [12] take an effective value $U-J=5 \mathrm{eV}$, where $J$ is the intra-atomic exchange coupling. Nicklas et al. [17] do not take any value of $U$ for Fe. As Lin and Jones [13] show, the actual value of $U$ greatly affects the computed exchange couplings, indeed, they find a difference of a factor of 2 between their calculations with $U=4.9 \mathrm{eV}$ and without $U$, finding $\mathrm{DFT}+U$ results in better agreement with the experiment.

In this work, we have used the $\mathrm{GGA}+U$ method of Dudarev [31] with a $U_{\text {eff }}=U-J$ of $1 \mathrm{eV}$ for Fe and $4 \mathrm{eV}$ for $\mathrm{Mn}$. The chosen values correspond to roughly subtracting $J \approx 1$ to $U=4.9 \mathrm{eV}(U=2 \mathrm{eV})$ as computed by Lin and Jones [13] for $\mathrm{Mn}(\mathrm{Fe})$ ontop a $\mathrm{Cu}$ atom. As we will see, the values of $U_{\text {eff }}$ become critical for the determination of the exchange couplings. The effect of different values for $U_{\text {eff }}$ is discussed below.

To rationalize our results, we have fitted them to a Heisenberg Hamiltonian in the form

$$
H=-\sum_{i, j>i} J_{i j} S_{i} \cdot S_{j},
$$

where $J_{i j}$ are the exchange couplings between spins $S_{i}$ and $S_{j}$. Evaluating different magnetic configurations we have been able to extract the $J_{i j}$ values by fitting the DFT energies to the Heisenberg Hamiltonian, Eq. (1).

For the shorter chains (FeMn and $\mathrm{FeMn}_{2}$ ), we have included spin-orbit coupling (SOC) [32,33] into our scalar-relativistic Hamiltonian. We have considered a simple anisotropic spin Hamiltonian, in the form

$$
H=D S_{i}^{2}+E\left(S_{j}^{2}-S_{k}^{2}\right) .
$$

Here, $i, j$, and $k$ are such that $i$ is the computed magnetic axis, while $j$ and $k$ are two orthogonal directions in the plane perpendicular to $i$ (hard-plane) in principle, different from the surface directions. The energy of the system has been self-consistently calculated including SOC for the different orientations $i, j$, and $k$ of the magnetization axis and, from these energies, the values for $D$ and $E$ in Eq. (2) have been fitted using the evaluated magnetic moments.

\section{RESULTS}

\section{A. Geometries and energetics}

Constant current images were obtained between atom manipulation sequences. This permitted us to have precise knowledge of the atomic arrangement. Unfortunately, the structure of the tip that is optimal for atom manipulation is not necessarily good for image production and the obtained images do not contain much information, as can be seen in Fig. 1. In the inset, we show the configuration as inferred from the atomic manipulation procedure that corresponds to a $\mathrm{FeMn}_{3}$ chain.

The calculation yields precise insight on the actual geometries. In Fig. 2, we show the FeMn 2 geometry as an example. As we can see in the side view, after deposition of the TM atoms the final chain includes the $\mathrm{N}$ atoms in between them. These atoms are lifted from the surface. $\mathrm{N}$ atoms at the edge of the chain, like $\mathrm{N}_{1}$, are moved upwards by $\approx 0.7 \AA$, while $\mathrm{N}$ atoms in between TM atoms, like $\mathrm{N}_{2}$, are lifted by $\approx 1.6 \AA$ with respect to their unrelaxed positions in the bare $\mathrm{CuN}_{2}$ surface. Therefore we can assume that the final chains have the form $\mathrm{FeMn}_{x} \mathrm{~N}_{x+2}$. Of course, this is just a conventional choice to 


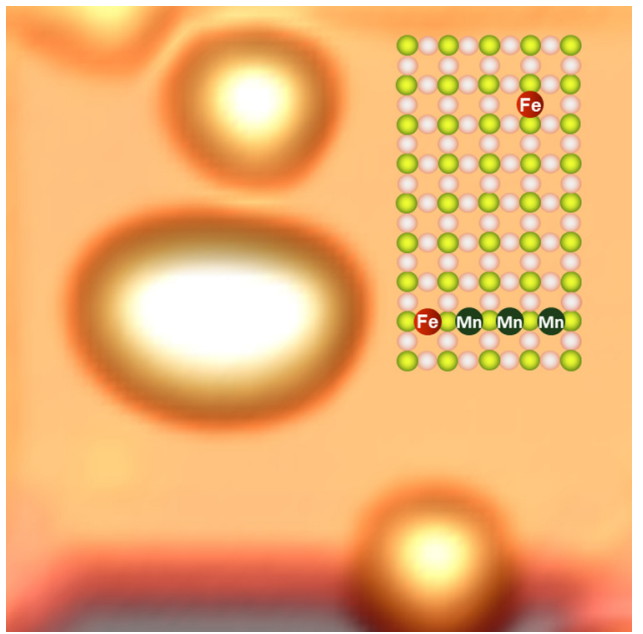

FIG. 1. Constant current STM image of $\mathrm{F} \mathrm{FeMn}_{3}$ chain assembled on a $\mathrm{Cu}_{2} \mathrm{~N}$ layer and atomic scheme (on the right of the figure) that can be inferred from the position of the upper-right Fe atom and manipulation with the STM tip. The STM image was scanned at $10 \mathrm{mV}$ and $100 \mathrm{pA}$. The scanned area is $6 \times 6 \mathrm{~nm}^{2}$. The transitionmetal atoms were always placed on top of a $\mathrm{Cu}$ atom between two nitrogen atoms. The topography was processed with WSXM [25].

identify the final chain, since other atoms in the surface are also significantly disturbed and could be considered as parts of the chain, like $\mathrm{Cu}_{\mathrm{S} 1}$ and $\mathrm{Cu}_{\mathrm{S} 2}$.
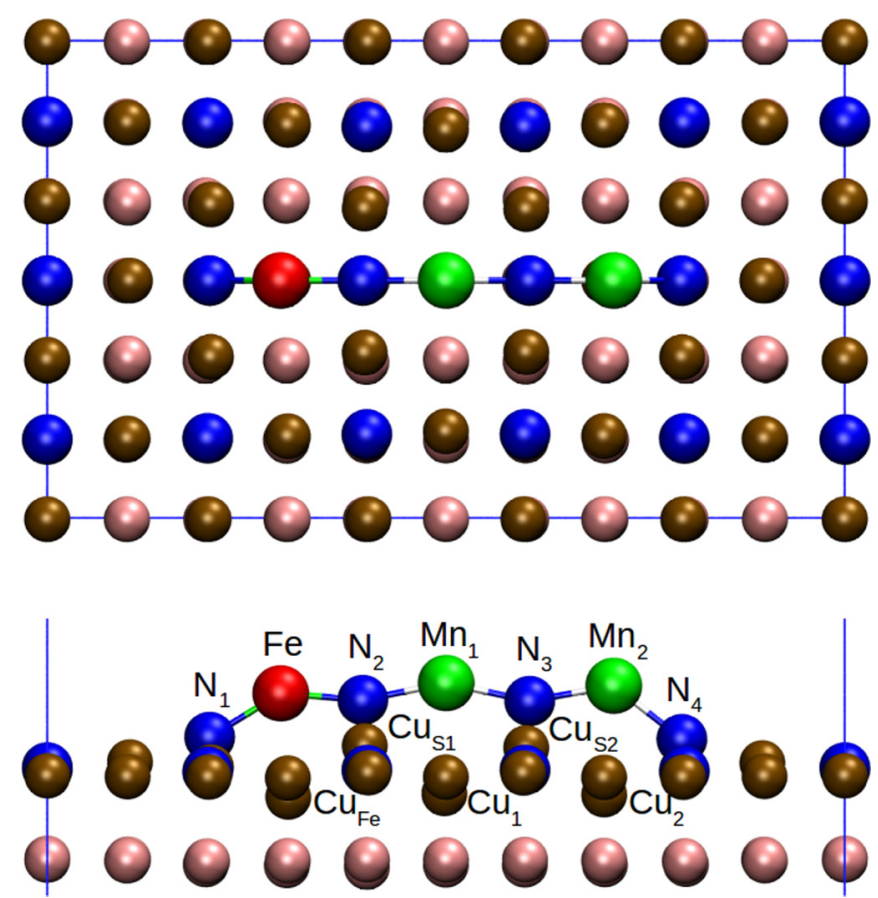

FIG. 2. Top (top panel) and side (bottom panel) views of the relaxed geometry of $\mathrm{FeMn}_{2}$. We show $\mathrm{Fe}$ atoms in red; Mn atoms in green; $\mathrm{N}$ atoms in blue; $\mathrm{Cu}$ atoms in the first layer in brown; the rest of $\mathrm{Cu}$ atoms in pink. Blue lines delimit the unit cell used in the calculations. The vacuum side of the unit cell and the three bottom $\mathrm{Cu}$ layers have been removed for pictorial reasons.
It is interesting to study the evolution of the geometry of the chains as their size increases. In Table I, we show the distance between TM atoms within the chain for all considered sizes. We observe that these distances are bigger than the unrelaxed value (3.65 $\AA$ corresponding to the PBE lattice parameter of $\mathrm{Cu}$ due to the arrangement of the chain on the surface). For example, $d\left[\mathrm{Fe}-\mathrm{Mn}_{1}\right]$ for FeMn is $3.81 \AA$, $4 \%$ bigger than the unrelaxed value. This distance tends to decrease as the chain size is increased, reaching $3.73 \AA$ for FeMn ${ }_{6}$. The same behavior can be observed for other positions in the chain. Another interesting distance to track involves the $\mathrm{Cu}$ atoms just underneath $\mathrm{Fe}$ and $\mathrm{Mn}$ atoms. As can be seen in the side view of Fig. 2, these $\mathrm{Cu}$ atoms $\left(\mathrm{Cu}_{\mathrm{Fe}}, \mathrm{Cu}_{1}, \mathrm{Cu}_{2}\right)$ are pushed downwards by around $0.4 \AA$. For FeMn, the $\mathrm{Fe}-\mathrm{Cu}_{\mathrm{Fe}}$ distance is very close to the value for the isolated atom $(2.37 \AA$ versus $2.38 \AA$ ). However, it increases for longer chains, with a value of $2.40 \AA$ for $\mathrm{FeMn}_{6}$. The same behavior is observed for the Mn atoms, again close to the single adsorbate for the case of FeMn $(2.50 \AA$ versus $2.47 \AA$ ), and it increases for longer chains reaching $2.56 \AA$ for $\mathrm{FeMn}_{6}$. Hence, as the number $x$ of $\mathrm{Mn}$ atoms increases, we find that the intra-chain distances diminish, see Table I, while the distance between the chain atom and the top $\mathrm{Cu}$ ones increases. Both behaviors can be rationalized as stress built up as the chain increases its size. Indeed, the elongation of these distances can be easily identified with some destabilization of the chains as length is increased.

To check for the stability of the chains we have studied the chain's energetics. First, we start by analyzing the atomization energy per TM atom, $E_{\text {at }}\left[\mathrm{FeMn}_{x}\right]$, defined from

$$
\begin{aligned}
E_{\mathrm{at}}\left[\mathrm{FeMn}_{x}\right]= & -\frac{1}{x+1}\left[E\left[\mathrm{FeMn}_{x}\right]-E[\mathrm{Fe}]\right. \\
& \left.-x E[\mathrm{Mn}]-E_{x}\left[\mathrm{Cu}_{2} \mathrm{~N}\right]\right],
\end{aligned}
$$

where $E\left[\mathrm{FeMn}_{x}\right]$ is the total energy of the system; $E[\mathrm{Fe}]$ $(E[\mathrm{Mn}])$ is the energy of a gas-phase $\mathrm{Fe}(\mathrm{Mn})$ atom; and $E_{x}\left[\mathrm{Cu}_{2} N\right]$ is the energy of the $[3 \times(x+3)]$ unit cell of the bare surface. The atomization energy per TM atom is a measure of how much average energy per atom one needs to give to the adsorbed chain to separate it in its constituent $\mathrm{Fe}$ and Mn gas-phase atoms and the pristine $\mathrm{Cu}_{2} \mathrm{~N} / \mathrm{Cu}(100)$ substrate. Figure 3 shows the atomization energy per TM atom as a function of the number of Mn atoms, $x$. The atomization energy per TM atom tends to decrease as the chain size increases, implying that long chains become energetically less favorable.

We can extract more information by looking at another quantity that reflects better the experimental procedure to construct the chain. Let us remember that the chains are constructed by STM manipulation. First, Fe and Mn atoms are deposited on the surface, and then they are moved with the STM tip to form the desired structure. To account for this procedure, we can define a formation energy where the reference of energy is the one of the deposited TM atom, and then to study the gain in energy by adding one more atom to the chain. The definition that we used for the formation energy is given by

$$
E_{F}\left[\mathrm{FeMn}_{x}\right]=-\left[E_{\mathrm{at}}\left[\mathrm{FeMn}_{x}\right]-E_{\mathrm{at}}[\mathrm{Fe}]-x E_{\mathrm{at}}[\mathrm{Mn}]\right],
$$

where we use the atomization energies defined in Eq. (3). From $E_{F}$, we can define the gain in energy by adding one more TM 
TABLE I. Distances between the different TM atoms for the six considered chains (in angstroms). Note that the unrelaxed value would be $3.65 \AA$ in agreement with the DFT lattice parameter of the $\mathrm{Cu}$ substrate.

\begin{tabular}{lccccc}
\hline \hline & $d\left[\mathrm{Fe}-\mathrm{Mn}_{1}\right]$ & $d\left[\mathrm{Mn}_{1}-\mathrm{Mn}_{2}\right]$ & $d\left[\mathrm{Mn}_{2}-\mathrm{Mn}_{3}\right]$ & $d\left[\mathrm{Mn}_{3}-\mathrm{Mn}_{4}\right]$ & $d\left[\mathrm{Mn}_{4}-\mathrm{Mn}_{5}\right]$ \\
\hline $\mathrm{FeMn}$ & 3.81 & & & & \\
$\mathrm{FeMn}_{2}$ & 3.77 & 3.84 & & & \\
$\mathrm{FeMn}_{3}$ & 3.75 & 3.80 & 3.82 & 3.79 & 3.77 \\
$\mathrm{FeMn}_{4}$ & 3.74 & 3.77 & 3.78 & 3.77 & 3.77 \\
$\mathrm{FeMn}_{5}$ & 3.73 & 3.75 & 3.76 & 3.74 & 3.74 \\
$\mathrm{FeMn}_{6}$ & 3.73 & 3.74 & 3.75 & $\left.\mathrm{Mn}_{6}\right]$ \\
\hline \hline
\end{tabular}

atom, $\Delta_{x} E_{F}[34]$, as

$$
\begin{aligned}
\Delta_{x} E_{F} & =E_{F}\left[\mathrm{FeMn}_{x}\right]-E_{F}\left[\mathrm{FeMn}_{x-1}\right] \\
& =-\left[E_{\mathrm{at}}\left[\mathrm{FeMn}_{x}\right]-E_{\mathrm{at}}\left[\mathrm{FeMn}_{x-1}\right]-E_{\mathrm{at}}[\mathrm{Mn}]\right] .
\end{aligned}
$$

This quantity, $\Delta_{x} E_{F}$, defines the energy gained by adding a $\mathrm{Mn}$ atom to an existing chain starting from an $\mathrm{Fe}$ atom, which mimics the experimental procedure to build the system. The calculated values of $\Delta_{x} E_{F}$ are always positive indicating that building the chains is an energetically favorable process. The energy gain is modest $(\approx 0.4 \mathrm{eV})$, and decreases by $37 \%$ from FeMn to $\mathrm{FeMn}_{6}$ (Fig. 3). Experimentally, the longest chains contained nine Mn atoms [23]. Our results seem to suggest that longer chains will be difficult to form due to the accumulated stress imposed by the surface lattice constant and the $\mathrm{Fe}-\mathrm{Mn}$ distance.

\section{B. Electronic and magnetic properties and charge transfer}

The magnetic properties are one of the main motivations for the study of these systems. They largely stem from the electronic structure of the atomic chains on the surface. An analysis of the electronic structure by projection onto atomic orbitals have been shown in Ref. [23]. The magnetic properties were shown to be given by the population of $\mathrm{Fe}$ and $\mathrm{Mn}$ $d$ orbitals. In the case of $\mathrm{Fe}$, the $d_{z}^{2}$ orbital was basically

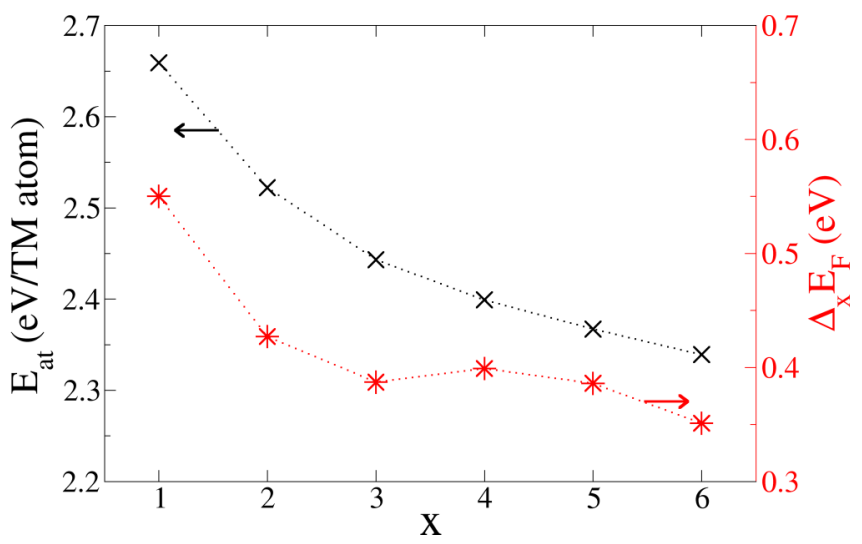

FIG. 3. Atomization energy, $E_{\text {at }}$ from Eq. (3), (black x's) and the gain in energy by adding one more $\mathrm{Mn}$ atom to the chain, $\Delta_{x} E_{F}$ from Eq. (4), (red stars). As we can see, atomization of the chain becomes less difficult as the chain becomes longer, and correspondingly, adding one more atom does not reduce the total energy as much for longer chains. These values reveal an increasing destabilization of the chain as it size increases. The dotted lines are a guide for the eye. filled (where $z$ is the direction perpendicular to the surface in the present case), not contributing to the atomic magnetic moment. The Mn atoms show a larger spin polarization due to the complete polarization of its $d$ shell after inclusion in the $\mathrm{FeMn}_{x}$ chain. The exchange splitting of $d$ bands is the smallest for $\mathrm{Fe}$, but still it is roughly $5 \mathrm{eV}$, showing how robust magnetism is in these chains. The electronic structure near the Fermi energy is largely due to the $\mathrm{Cu}$ and $\mathrm{N}$ states and with negligible magnetic polarization.

It is interesting to inspect the total magnetic moment of the chains shown in Table II. We can see how the magnetic couplings between Fe-Mn and $\mathrm{Mn}-\mathrm{Mn}$ atoms are antiferromagnetic (AF) in the most stable configuration. In addition, the magnetic moment of atomic Fe on the surface is $3.35 \mu_{B}$ (formally $S=2$ ), while the value for $\mathrm{Mn}$ is $4.80 \mu_{B}$ (formally $S=\frac{5}{2}$ ). Therefore formally, when adding Mn atoms to a Fe one, we would get systems with $S=\frac{1}{2}$ for an odd number of $\mathrm{Mn}$ atoms, $x$, and $\mathrm{S}=2$ for an even number. In the computed results for the total magnetic moment in the cell $\left(\mu_{T}\right)$ we observe the expected even-odd behavior, with values of 1.30-1.43 Bohr magnetons, $\mu_{B}$, for $x$ odd, and 3.68-3.86 $\mu_{B}$ for the $x$ even. Values of $1.30-1.43 \mu_{B}$ correspond well with the simple result $S=\frac{1}{2}$. The difference from $1 \mu_{B}$ has two different sources that are present in these calculations. On the one hand, the TM atoms present fractional occupancies that lead to noninteger multiples of $\mu_{B}$. On the other hand, these calculations are mean-field approximations to the difficult solutions of correlated AF ground states. As a consequence, the mean-field solution averages over the possible atomic magnetic moments found in the multiple spin configurations of the correct AF ground state.

The AF coupling between the TM atoms is mediated by the $\mathrm{N}$ atoms via a superexchange mechanism as previously shown for $\mathrm{Mn}$ [12] and Fe chains [17]. To illustrate the superexchange interaction, we show the spin-difference-density of the FeMn 3 chain in Fig. 4. The spin polarization of the intercalated $\mathrm{N}$ atoms adopts the expected form for superexchange, with induced spin polarization within the atom, but net spin close to zero. Indeed, the bigger values for the induced magnetic moments of $\mathrm{N}$ atoms are very small, for example $0.11 \mu_{B}$ for $\mathrm{N}_{1}$ and $0.08 \mu_{B}$ for $\mathrm{N}_{2}$. Superexchange also leads to a change in the atomic angles: the Fe-N-Mn and Mn-N-Mn angles tend to approach $180^{\circ}$ maximizing the AF interactions [12,17].

The magnetic moments tend to increase with increasing chain size, which is consistent with the previously mentioned progressive separation of the chain from the surface. The magnetic moments of the $\mathrm{Mn}$ atoms within a given chain are 
TABLE II. Magnetic moments (in $\mu_{B}$ ) of the TM atoms in the chains. Values for isolated Fe and Mn atoms on the surface are included for comparison. The values have been obtained by performing a Bader analysis [30]. The total magnetic moment $\mu_{T}$ of the calculation cell also includes contributions from nearby copper atoms that spin polarize, hence $\mu_{T}$ slightly differs from the sum of the magnetic moments of Fe and Mn.

\begin{tabular}{|c|c|c|c|c|c|c|c|c|}
\hline & $\mu_{T}$ & $\mu[\mathrm{Fe}]$ & $\mu\left[\mathrm{Mn}_{1}\right]$ & $\mu\left[\mathrm{Mn}_{2}\right]$ & $\mu\left[\mathrm{Mn}_{3}\right]$ & $\mu\left[\mathrm{Mn}_{4}\right]$ & $\mu\left[\mathrm{Mn}_{5}\right]$ & $\mu\left[\mathrm{Mn}_{6}\right]$ \\
\hline $\mathrm{Fe}$ & 3.82 & 3.35 & & & & & & \\
\hline Mn & 4.97 & & 4.80 & & & & & \\
\hline FeMn & -1.30 & 3.16 & -4.65 & & & & & \\
\hline $\mathrm{FeMn}_{2}$ & 3.68 & 3.17 & -4.55 & 4.71 & & & & \\
\hline $\mathrm{FeMn}_{3}$ & -1.43 & 3.19 & -4.56 & 4.56 & -4.73 & & & \\
\hline $\mathrm{FeMn}_{4}$ & 3.78 & 3.22 & -4.56 & 4.57 & -4.57 & 4.75 & & \\
\hline $\mathrm{FeMn}_{5}$ & -1.36 & 3.23 & -4.56 & 4.58 & -4.58 & 4.58 & -4.77 & \\
\hline $\mathrm{FeMn}_{6}$ & 3.86 & 3.23 & -4.57 & 4.58 & -4.58 & 4.59 & -4.58 & 4.79 \\
\hline
\end{tabular}

quite constant, with the exception of the final atom, which is $\approx 4 \%$ bigger. The reason is the lower coordination of the edge atom, reflected in the slightly longer distance with the $\mathrm{Cu}$ atom below and the longer distance with the adjacent $\mathrm{N}$ atoms.

The analysis of charge transfer in the system yields that each TM atom in the chain loses around one $s$ electron to form the bond with $\mathrm{N}$ atoms in the chain and the $\mathrm{Cu}$ atom underneath the TM atom. The $d$ charge is similar to the atomic case, i.e., there are six electrons in the $d$ manifold for $\mathrm{Fe}$, and five for $\mathrm{Mn}$, in agreement with the computed magnetic moments.

\section{Exchange coupling constants}

We have fitted our results to the Heisenberg Hamiltonian shown in Eq. (1). In order to do so, we have calculated different spin solutions corresponding to different magnetic ordering among the TM atoms in the chain. In all calculations, we have used the geometry of the most stable configuration, which is always the AF case shown in Table II. For the atomic values of $S$, in Eq. (1), we had the choice of either using the formal value ( $S=2$ for Fe, $S=\frac{5}{2}$ for $\mathrm{Mn}$ ), or taking the computed value from the DFT calculation since for a single atom $S_{z}$ is a good quantum number. We have opted for the later option, which gives values for the exchange coupling constants $J$ 's, which are between $15 \%$ and $20 \%$ higher. We can justify this choice because the DFT values reflect the local magnetic moment that interacts via other atoms (superexchange) with the local magnetic moment of the next neighbor in the calculation. The results are shown in Table III. As expected, $J$ 's involving first neighbors are negative, which indicate an AF coupling. Second

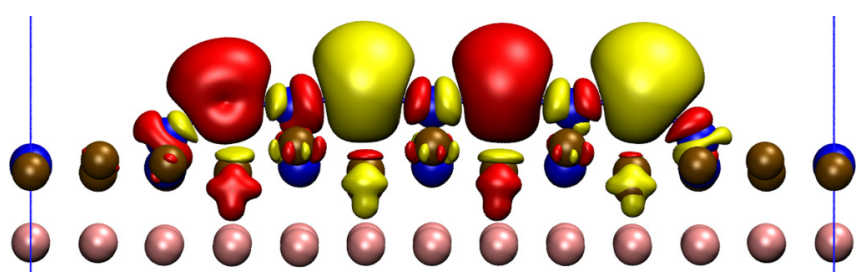

FIG. 4. Spin density of the FeMn 3 chain. Red (yellow) indicates majority (minority) spin areas. The chosen isovalue is $0.01 \mathrm{eV} / \AA^{3}$. The color code of the atoms is the same of Fig. 2. neighbor $J$ 's are positive, indicating a ferromagnetic coupling, which further stabilizes the global AF solution. Further order J's are smaller, and its omission in the fit only implies a small error.

The convergence with the number of coupled atoms considered in the Heisenberg chain, Eq. (1), is very fast. We have done a systematic study for $\mathrm{FeMn}_{4}$ where different number of neighbors are included in solving Eq. (1). Considering just first neighbors introduces a maximum error of $0.2 \mathrm{meV}$. Considering first and second neighbors the error is less than $0.1 \mathrm{meV}$. These calculations show that the effective interactions in these spin chains are very short range and first-neighbors truncation is indeed an accurate approximation.

Analyzing the evaluated exchange couplings, $J$, we observe that the biggest value is obtained for the first-neighbors interaction between $\mathrm{Fe}$ and $\mathrm{Mn}_{1}$. Thus the $\mathrm{AF}$ coupling between Fe and $\mathrm{Mn}$ is stronger than the one between $\mathrm{Mn}-\mathrm{Mn}$. FeMn is a special case since it involves two edge atoms, the resulting AF coupling is the strongest one. Curiously, the exchange coupling between $\mathrm{Fe}$ and $\mathrm{Mn}_{1}\left(J_{12}\right)$ presents a sharp drop for $\mathrm{FeMn}_{2}$ to start increasing again for $\mathrm{FeMn}_{3}$ and $\mathrm{FeMn}_{4}$. The coupling between $\mathrm{Mn}_{1}$ and $\mathrm{Mn}_{2}\left(J_{23}\right)$ presents the same behavior, it shows a minimum for FeMn $\mathrm{F}_{3}$. Similar behavior is obtained for pure Mn chains by Rudenko et al. [12]. These minima in the exchange couplings seem to be independent from the atomic geometry, where the behavior with chain length is monotonous, and with the atomic magnetic moments. It is probably due to the sudden appearance of an extra neighbor that symmetrizes the interactions on the central atom of the chain.

As Rudenko et al. [12] and Lin and Jones [13] note, there is a dependence of the values of the interatomic exchange coupling on the chosen value of $U_{\text {eff }}=U-J$ for the GGA $+U$ approximation. Figure 5 shows the dependence of $J_{12}$ for FeMn on $U_{\text {eff }}$. We find that as the value of $U_{\text {eff }}$ decreases, $\left|J_{12}\right|$ increases. For the values of the present study [13] $\left(U_{\text {eff }}\right.$ $[\mathrm{Fe}]=1 \mathrm{eV}$ and $\left.U_{\text {eff }}[\mathrm{Mn}]=4 \mathrm{eV}\right) J_{12}=-10.25 \mathrm{meV}$. If $U_{\text {eff }}[\mathrm{Fe}]$ is reduced by $1 \mathrm{eV}$, this value changes to -11.60 meV. When $U_{\text {eff }}[\mathrm{Mn}]$ is reduced by $1 \mathrm{eV}$ the effect is more pronounced, obtaining a value of $-16.44 \mathrm{meV}$ (60\% increase in absolute value). These values give us an interval of exchange couplings that will be compared with our experimental results in the following sections. 
TABLE III. Values for the exchange coupling constants (in meV) obtained by fitting Eq. (1) to the GGA $+U$ total energies.

\begin{tabular}{|c|c|c|c|c|c|c|c|c|c|c|}
\hline & \multicolumn{4}{|c|}{ First neighbors } & \multicolumn{3}{|c|}{ Second neighbors } & \multicolumn{2}{|c|}{ Third neighbors } & \multirow{2}{*}{$\frac{\text { Fourth }}{J_{15}}$} \\
\hline & $J_{12}$ & $J_{23}$ & $J_{34}$ & $J_{45}$ & $J_{13}$ & $J_{24}$ & $J_{35}$ & $J_{14}$ & $J_{25}$ & \\
\hline FeMn & -10.25 & & & & & & & & & \\
\hline $\mathrm{FeMn}_{2}$ & -6.51 & -2.09 & & & 0.97 & & & & & \\
\hline $\mathrm{FeMn}_{3}$ & -7.30 & -1.97 & -1.97 & & 1.14 & 0.32 & & 0.10 & & \\
\hline $\mathrm{FeMn}_{4}$ & -7.96 & -2.69 & -2.10 & -2.16 & 0.73 & 0.05 & 0.27 & -0.36 & -0.01 & -0.02 \\
\hline
\end{tabular}

\section{Magnetic anisotropy}

We have studied the magnetic anisotropy energy (MAE) of the system by including SOC in our calculations. We have performed self-consistent calculations in three directions: along the chain $(y)$, perpendicular to the chain in the plane $(x)$, and perpendicular to the plane $(z)$.

We have also fitted the anisotropic spin Hamiltonian of Eq. (2) to the singly adsorbed atoms using their computed magnetic moments to obtain values for $D$ and $E$ using the equations:

$$
\begin{aligned}
& D=\frac{2 E_{i}-\left(E_{j}+E_{k}\right)}{S(2 S-1)}, \\
& E=\frac{E_{j}-E_{k}}{S(2 S-1)},
\end{aligned}
$$

where $E_{i}, E_{j}$, and $E_{k}$ are the magnetic anisotropy energies when the spin, $S$, is aligned along the easy or hard axis $i$ and the two transversal axes $j$ and $k$, see Table IV.

For $\mathrm{Fe}$, the minimun of MAE lies along the $\mathrm{N}$ direction, $i=y$, with a value of $1.88 \mathrm{meV}$ in good agreement with previous calculations $[35,36]$. The transversal direction show larger values of the energy as compared with previous calculations [35,36]. For Mn, the MAE is much smaller and with a value of $0.15 \mathrm{meV}$ in good agreement with Ref. [35].

Our results have been tested against convergence with unit cell size [37] and present a negligible dependence. Moreover, MAE calculations are very robust against different DFT approximations. Using GGA $+U$, Barral et al. [36] obtained similar results to the strictly LDA calculations by Shick

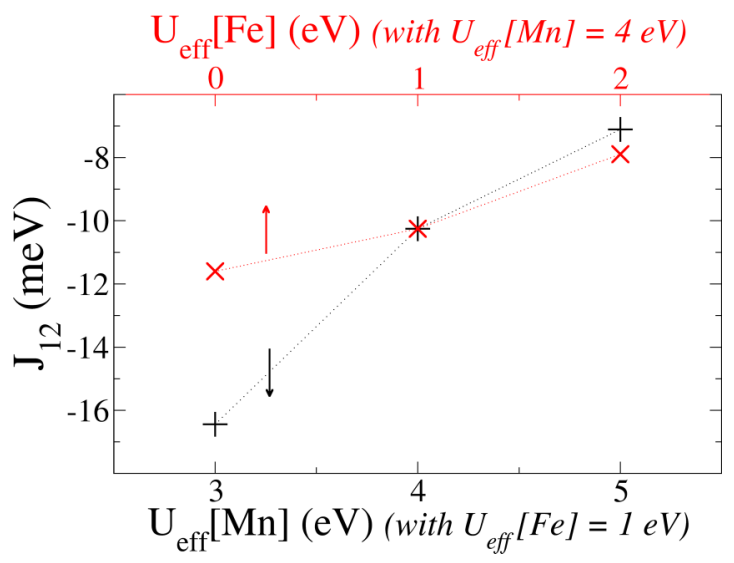

FIG. 5. Evolution of $J_{12}$ for FeMn with respect to the variation of $U_{\text {eff. }}$. We have changed one of the values of $U$ while keeping constant the other one. et al. [35]. Panda et al. [38] obtain a small change in D when they use an extraordinarily large value of $U$ (from $D=-0.48 \mathrm{meV}$ for $U=0$ to $D=-0.67 \mathrm{meV}$ for $U=6$ eV). Hence, as Panda et al. [38] show, improved calculations of MAE need to go beyond DFT.

The calculation of $D$ and $E$ differ from reported values. Our calculations compared better with the values of Ref. [35] if the nominal magnetic moments are used. Indeed, we obtain $D=-0.44 \mathrm{meV}$ and $E=0.19 \mathrm{meV}$ using $S=2$ for Fe and $D=-0.67 \mathrm{meV}, E=0.29 \mathrm{meV}$ for the computed magnetic moments. These values are obtained if we assume an easy axis along the $y$ direction as found experimentally [22]. For Mn the agreement with Ref. [35] improves probably because the DFT magnetic moment agrees with the nominal one. However, Barral et al. [36] report on a different spin direction for Mn. As these last authors discuss, the MAE values for Mn on $\mathrm{Cu}_{2} \mathrm{~N}$ are very small and prone to numerical inaccuracy. These results support the idea that MAE calculations require postDFT treatments such as the very recent ones of Ref. [38].

Taking into account that experimentally $\mathrm{Mn}$ and Fe display perpendicular easy axes [22], one may wonder if the SOC might induce a noncollinear alignment of the magnetic moments in the chains. We have tested that possibility for the shorter chains by performing an explicit noncollinear calculation including SOC [33]. Due to the small energies involved in the calculation, the convergence of noncollinear solutions is very challenging. We have been able to stabilize a noncollinear configuration for FeMn where the magnetic moment of $\mathrm{Fe}$ is along the chain, while the moment for $\mathrm{Mn}$ forms an angle of $27^{\circ}$ with the $y$ axis. However, this solution is $3.79 \mathrm{meV}$ higher in energy than the collinear solution. For longer chains, we have not been able to stabilize any noncollinear solution. Therefore we have just considered collinear configurations for the rest of our calculations.

The larger MAE of $\mathrm{Fe}$ forces the full-chain magnetic axis to align with the $\mathrm{Fe}$ anisotropy axis. This is indeed

TABLE IV. Magnetic anisotropy energies (MAE) (in meV). We also show the easy axis directions, where $x$ is the direction in plane and perpendicular to the chain, $y$ along the chain, and $z$ perpendicular to the surface.

\begin{tabular}{lcrr}
\hline \hline MAE $(\mathrm{meV})$ & dir & $E_{z}-E_{y}$ & $E_{x}-E_{y}$ \\
\hline $\mathrm{Fe}$ & $y$ & 0.74 & 1.88 \\
$\mathrm{Mn}$ & $z$ & -0.15 & -0.02 \\
$\mathrm{FeMn}$ & $y$ & 0.75 & 1.51 \\
$\mathrm{FeMn}$ & $y$ & 0.48 & 1.46 \\
\hline \hline
\end{tabular}


seen in Table IV, where the value for MAE decreases when increasing the chain length. This reduction in MAE can be understood in terms of the addition of $\mathrm{Mn}$ atoms that tend to align their magnetic axis perpendicular to the $\mathrm{Fe}$ one. These results qualitatively agree with the results of anisotropic spin Hamiltonians where the total magnetic anisotropy is approximated by adding the individual contributions of $D$ and $E$. This approximation does not capture the changes in geometry of the chain with increasing lentgth. Nevertheless, adding up the MAE contributions of each atom leads to overestimations of MAE that are below the accuracy of our DFT calculations.

The study of the magnetic anisotropy in these chains reveals their composite structure. All FeMn ${ }_{x}$ for odd- $x$ show a quasispin of $1 / 2$, which should present zero anisotropy if these chains were macrospins of spin 1/2, see. Eq. (2). Our DFT calculations show sizable anisotropies that underscore the complexity of the magnetic states of these antiferromagnetic structures.

\section{E. Magnetic excitation energies}

The magnetic structure of the chains can be obtained by studying the inelastic electron tunneling spectra (IETS) [9] obtained with the STM. Figure 6 shows the differential conductance obtained for the different $\mathrm{FeMn}_{x}$ chains. The features that appear in these spectra are due to magnetic excitations, very similarly to the ones of $\mathrm{Mn}_{x}$ chains shown in Ref. [22]. Nevertheless, there are noticeable differences regarding both the peak at zero bias for the odd- $x$ chains and the detailed structure of the steps. Indeed, even- $x$ chains are singlets in the case of Mn chains, here, however, the ground states of odd- $x$ chains present a doublet $\left(S \approx \frac{1}{2}\right)$ magnetic structure.

We solve Eq. (1) and study the different magnetic states for each chain using the methodology of Ref. [39]. The study of the ground state is particularly important to understand the behavior of the Kondo physics appearing in Fig. 6 [23].

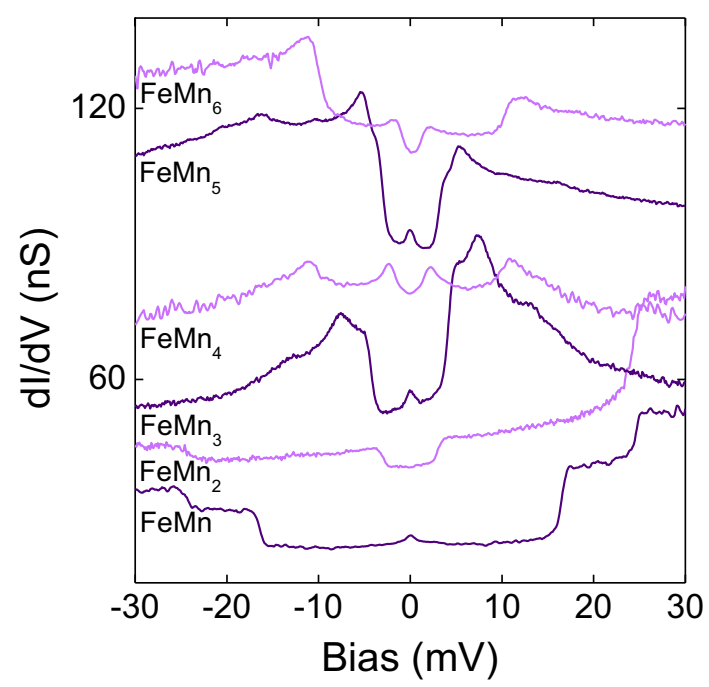

FIG. 6. Inelastic electron tunneling spectra (IETS) of different $\mathrm{FeMn}_{x}$ chains obtained by measuring the differential conductance with the STM tip placed on a central Mn atom.
Indeed, all odd- $x$ chains display a Kondo zero-bias peak, while this peak is absent from the chains with even $x$. Due to the Heisenberg correlations, the experimental ground state is multi-configurational. Solving Eq. (1) with the anisotropy terms, Eq. (2) shows that many of these configurations are $S=\frac{1}{2}$ states, with a weight larger than $20 \%$ in the total state. Hence a spin-flip process is possible at zero-energy cost, which explains the appearance of Kondo peaks. Likewise, even- $x$ chains do not have degenerate ground states and Kondo physics is absent. The absence of degenerate ground states for even- $x$ is due to a total spin $S \approx 2$ where the large longitudinal MAE of the Fe atom lifts the degeneracy of the ground state. The presence of anisotropy prevents $S$ from being a good quantum number [23].

The solutions of Eq. (1) permit us to compare the computed data for the chains with the experimental data. Figure 7(a) shows the IETS for the FeMn 3 chain measured of the edge Mn atom, and Fig. 7(b) shows two calculations. The first one (blue) performed with the exchange couplings computed from our GGA $+U$ calculations with an effective $U$ value for $\mathrm{Mn}$ of $4 \mathrm{eV}$ and for $\mathrm{Fe}$ of $1 \mathrm{eV}$, see Sec. IV C, and adding up the experimental atomic magnetic anisotropies. Unfortunately, DFT seems to underestimate the anisotropy of $\mathrm{Fe}$ on $\mathrm{Cu}_{2} \mathrm{~N}$, see Sec. IV D, therefore we use the experimental values for the anisotropy. The second one (green) scales the exchange couplings by 1.6, using the scaling found for FeMn when the effective $U$ value for $\mathrm{Mn}$ was reduced to $3 \mathrm{eV}$, and keeping the corresponding value of Fe constant. We see that the energy thresholds are in good agreement in the second case, and the solution of Eq. (1) with the third-order perturbation method of Ref. [39] largely reproduces the dynamical phenomenology of the magnetic chain including higher-energy excitations like the one at $\pm 10 \mathrm{meV}$.

The asymmetry of the main inelastic thresholds found in Fig. 7(a) is treated within the third-order perturbation method by including a potential scattering term in the Kondo scattering [39]. The effect of the potential scattering term is to remove the electron-hole symmetry of the excitation spectra of Eq. (1). In order to fit the experimental spectra, we have used a $J_{\text {Kondo }} \rho=-0.04$ (where $J_{\text {Kondo }}$ is the Kondo exchange coupling with electrons from the substrate and $\rho$ is the density of states at the Fermi energy) and a potential scattering term $U_{\text {Kondo }} / J_{\text {Kondo }}=-0.5$. The results indicate that larger values should be used to reproduce the experimental data, implying the need to go beyond third-order perturbation theory to treat Kondo scattering in FeMn 3 .

The magnetic behavior of the inelastic thresholds is correctly reproduced by a Zeeman shift. This permits us to extract the value of the gyromagnetic ratio $g$, Fig. 8 . For the present case we find that the atomic g's $\left(g_{\mathrm{Fe}}=2.1\right.$ and $g_{\mathrm{Mn}}=1.9$ from Ref. [22]) are good approximations to obtain the correct behavior of the magnetic global states with external $B$, Fig. 8. To a large extend, the atomic spin preserves its character, although very entangled due to the sizable Heisenberg exchange interactions.

\section{DISCUSSION AND CONCLUDING REMARKS}

This work is a detailed experimental and theoretical account of the electronic and magnetic properties of a heterogeneous 


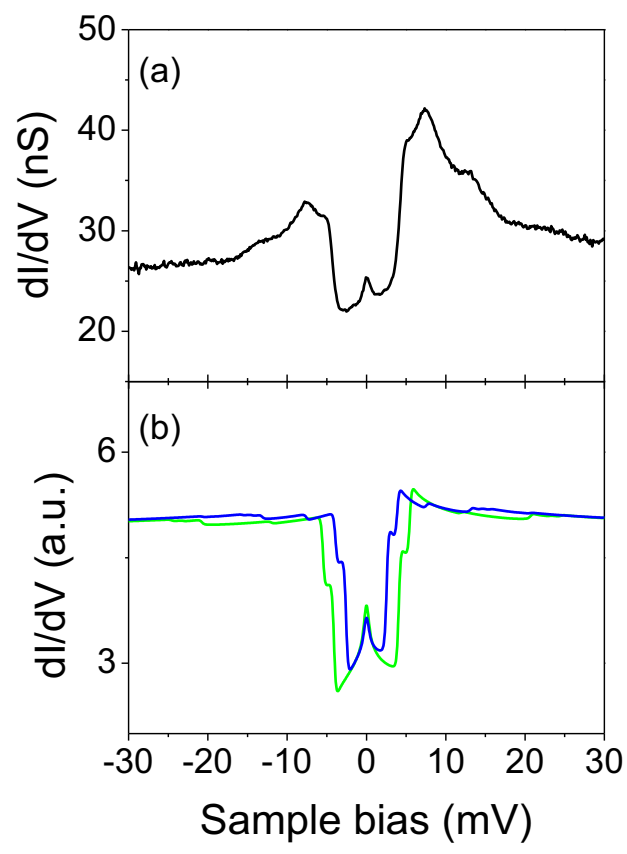

FIG. 7. Inelastic electron tunneling spectra (IETS) of $\mathrm{FeMn}_{3}$ : (a) experimental and (b) two computed solution using our calculated spin-chain parameters for $(U-J)_{\mathrm{Mn}}=4 \mathrm{eV}$ and $(U-J)_{\mathrm{Fe}}=1 \mathrm{eV}$ (blue) or scaling the computed exchange coupling by a 1.6 factor as found for FeMn with $(U-J)_{\mathrm{Mn}}=3 \mathrm{eV}$ and $(U-J)_{\mathrm{Fe}}=1 \mathrm{eV}$ (green), Sec. IV C. The STM tip is placed on the edge Mn atom.

type of magnetic atomic chain $\left(\mathrm{FeMn}_{x}\right.$ with $\left.x=1,6\right)$ adsorbed on $\mathrm{Cu}_{2} \mathrm{~N} / \mathrm{Cu}$ (100). The chains are assembled by atom manipulation with an STM tip, and stable configurations are found when the $\mathrm{TM}$ atoms $(\mathrm{TM}=\mathrm{Fe}$ and $\mathrm{Mn}$ ) sit on top of $\mathrm{Cu}$ atoms. Experimentally, it is difficult to assemble chains with $x>9$ (we rarely succeeded going beyond $x=10$ creating a straight $\mathrm{FeMn}_{x}$ chain) and theoretically we see that stress builds up as the chain increases size due to the imposed TM-TM distance by the underlying $\mathrm{Cu}_{2} \mathrm{~N} / \mathrm{Cu}$ (100) substrate. As the chain increases its size, the TM atoms increase their mutual distance and also their distance to the chain, energetically this is translated into a systematic lowering of both the atomization energy and the energy gained by the chain every time a new Mn atom is added.

Upon adsorption, $\mathrm{Fe}$ and $\mathrm{Mn}$ lose one of their $s$ electrons in the interaction with the substrate, mainly to form the bond with the neighboring $\mathrm{N}$ atoms. There is considerable distortion and hybridization of the d-electron structure but their occupations remain the free-atom ones, leading to magnetic moment values close to the gas phase $[13,22]$.

The experimental data involve the IETS of different chains with detailed information on the excitation energies of the chains. The lower-energy spectral features are due to magnetic excitations of the system as was tested by their magnetic field dependence. In parallel, the values of the Heisenberg Hamiltonian can be obtained from DFT by evaluating the energy of different spin arrangements of the chains. This approach gives us a systematic insight on how the different TM atoms relate to each other in the chain, which are mainly driven by antiferromagnetic superexchange mediated by the nonmagnetic $\mathrm{N}$ atoms of the surface. We find that the Fe-Mn couplings are systematically larger than the Mn-Mn ones, and that beyond second neighbors neglection of the magnetic coupling is a very good approximation. Indeed, first neighbors is a sufficient approximation to obtain exchange couplings with an error of $0.2 \mathrm{meV}$. In the present choice of DFT $+U$ calculations, the computed magnetic exchanges lead to excitation energies smaller than the experimental ones. Unfortunately, there is not a unique way of determining the value of the Hubbard $U$ for the calculations. Our systematic study of the values show that a change of $1 \mathrm{eV}$ in the value of the $\mathrm{Mn} U$ leads to a $\sim 60 \%$ change in the value of the evaluated couplings yielding good agreement with the experiment. Despite this dependence of the interatomic exchange energies on the value of $U$, the conclusions of this work do not change for the range of used values. Moreover, the geometrical, stability and magnetic anisotropy results show a negligible dependence with the above values of $U$.

The TM atoms are subjected to magnetic anisotropies on this surface. Our calculations show that the MAE of the full chain is not just the sum of the MAE's of each TM atom. Nevertheless, the smallness of Mn MAE renders this approximation acceptable. Despite their different magnetic axis, the very large MAE of the Fe dominates and the Mn
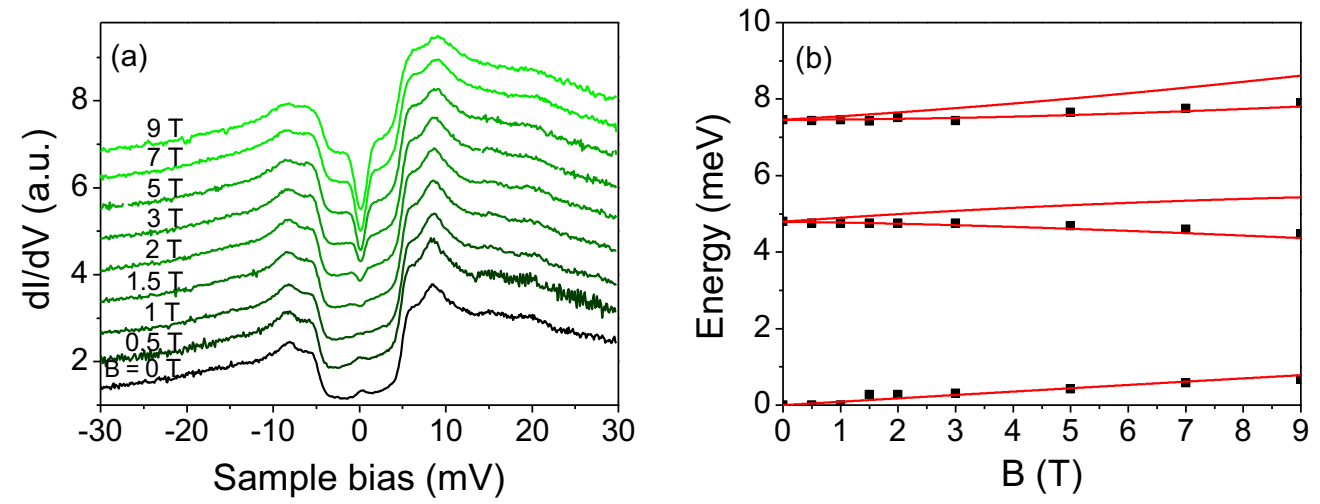

FIG. 8. (a) Inelastic electron tunneling spectra (IETS) for $\mathrm{FeMn}_{3}$ with a magnetic field applied perpendicular to the surface increasing until $9 \mathrm{~T}$. (b) The behavior of the different magnetic states (black dots) obtained from the experimental figure follows the Zeeman trend expected for a Zeeman term with the atomic $g$ factors $\left(g_{\mathrm{Fe}}=2.1\right.$ and $g_{\mathrm{Mn}}=1.9$ from Ref. [22]). 
spins orient along the chain following the Fe anisotropy axis. This leads to a collinear solution of the initially noncollinear problem. We have not found any spin canting or frustration although we cannot rule it out for longer chains. Moreover, the study of the magnetic anisotropy in these chains reveals their composite structure. All FeMn $\mathrm{M}_{x}$ for odd- $x$ show a quasi-spin of $1 / 2$. If these chains were spins $1 / 2$, their anisotropy would be strictly zero. However, our calculations show that they have sizable anisotropies in agreement with the complexity of the magnetic states of these antiferromagnetic structures.

This combined experimental and theoretical work gives us direct insight into the different electronic, geometric and magnetic properties of these heterogeneous chains. In particular, we have given an account for the appearance of Kondo peaks and the antiferromagnetic character of these chains, their magnetic anisotropy that permits us to rule out a macrospin behavior, as well as the accumulated stress that limits the length of the chains.

\section{ACKNOWLEDGMENTS}

D.-J.C. acknowledges the European Union for support under the H2020-MSCA-IF-2014 Marie-Curie Individual Fellowship programme Proposal No. 654469 and a previous postdoctoral fellowhship from the Alexander von Humboldt foundation. D.-J.C. and S.L. acknowledge Edgar Weckert and Helmut Dosch (Deutsches Elektronen-Synchrotron, Hamburg, Germany) for providing high-stability laboratory space. N.L. acknowledges financial support from Spanish MINECO (Grant No. MAT2015-66888-C3-2-R). ICN2 acknowledges support from the Severo Ochoa Program (MINECO, Grant No. SEV-2013-0295).
[1] H. Wang, Y. Yu, Y. Sun, and Q. Chen, NANO: Brief Rep. Rev. 6, 1 (2011).

[2] H. Brune, Science 312, 1005 (2006).

[3] C. F. Hirjibehedin, C. P. Lutz, and A. J. Heinrich, Science 312, 1021 (2006).

[4] A. A. Khajetoorians, J. Wiebe, B. Chilian, S. Lounis, S. Blügel, and R. Wiesendanger, Nat. Phys. 8, 497 (2012).

[5] S. Loth, S. Baumann, C. P. Lutz, D. M. Eigler, and A. J. Heinrich, Science 335, 196 (2012).

[6] S. Holzberger, T. Schuh, S. Blügel, S. Lounis, and W. Wulfhekel, Phys. Rev. Lett. 110, 157206 (2013).

[7] S. Yan, D.-J. Choi, J. A. J. Burgess, S. Rolf-Pissarczyk, and S. Loth, Nat. Nanotechnol. 10, 40 (2015).

[8] R. Wiesendanger, Rev. Mod. Phys. 81, 1495 (2009).

[9] A. J. Heinrich, J. A. Gupta, C. P. Lutz, and D. M. Eigler, Science 306, 466 (2004).

[10] J.-P. Gauyacq, N. Lorente, and F. Dutilh Novaes, Prog. Surf. Sci. 87, 63 (2012).

[11] S. Lounis, P. H. Dederichs, and S. Blügel, Phys. Rev. Lett. 101, 107204 (2008).

[12] A. N. Rudenko, V. V. Mazurenko, V. I. Anisimov, and A. I. Lichtenstein, Phys. Rev. B 79, 144418 (2009).

[13] C.-Y. Lin and B. A. Jones, Phys. Rev. B 83, 014413 (2011).

[14] M. C. Urdaniz, M. A. Barral, and A. M. Llois, Phys. Rev. B 86, 245416 (2012).

[15] K. Tao, Q. Guo, P. Jena, D. Xue, and V. S. Stepanyuk, Phys. Chem. Chem. Phys. 17, 26302 (2015).

[16] D.-J. Choi, R. Robles, J.-P. Gauyacq, C. Rubio-Verdú, N. Lorente, and J. I. Pascual, J. Phys.: Condens. Matter 28, 23LT01 (2016).

[17] J. W. Nicklas, A. Wadehra, and J. W. Wilkins, J. Appl. Phys. 110, 123915 (2011)

[18] J.-P. Gauyacq, S. M. Yaro, X. Cartoixà, and N. Lorente, Phys. Rev. Lett. 110, 087201 (2013).

[19] A. A. Khajetoorians, B. Baxevanis, C. Hübner, T. Schlenk, S. Krause, T. O. Wehling, S. Lounis, A. Lichtenstein, D. Pfannkuche, J. Wiebe, and R. Wiesendanger, Science 339, 55 (2013).
[20] A. Spinelli, B. Bryant, F. Delgado, J. Fernández-Rossier, and A. F. Otte, Nat. Mater. 13, 782 (2014).

[21] A. Spinelli, M. P. Rebergen, and A. F. Otte, J. Phys.: Condens. Matter 27, 243203 (2015).

[22] C. F. Hirjibehedin, C.-Y. Lin, A. F. Otte, M. Ternes, C. P. Lutz, B. A. Jones, and A. J. Heinrich, Science 317, 1199 (2007).

[23] D.-J. Choi, R. Robles, S. Yan, J. A. J. Burgess, S. RolfPissarczyk, J.-P. Gauyacq, N. Lorente, M. Ternes, and S. Loth, arXiv:1507.04785 [cond-mat.mes-hall].

[24] M. Kepenekian, J.-P. Gauyacq, and N. Lorente, J. Phys.: Condens. Matter 26, 104203 (2014).

[25] I. Horcas, R. Fernández, J. M. Gómez-Rodríguez, J. Colchero, J. Gómez-Herrero, and A. M. Barò, Rev. Sci. Instrum. 78, 013705 (2007).

[26] G. Kresse and J. Furthmüller, Comput. Mater. Sci. 6, 15 (1996).

[27] P. E. Blöchl, Phys. Rev. B 50, 17953 (1994).

[28] G. Kresse and D. Joubert, Phys. Rev. B 59, 1758 (1999).

[29] J. P. Perdew, K. Burke, and M. Ernzerhof, Phys. Rev. Lett. 77, 3865 (1996).

[30] W. Tang, E. Sanville, and G. Henkelman, J. Phys.: Condens. Matter 21, 084204 (2009).

[31] S. L. Dudarev, G. A. Botton, S. Y. Savrasov, C. J. Humphreys, and A. P. Sutton, Phys. Rev. B 57, 1505 (1998).

[32] G. Kresse and O. Lebacq, VASP Manual, http://cms.mpi.univie.ac.at/vasp/vasp/vasp.html.

[33] D. Hobbs, G. Kresse, and J. Hafner, Phys. Rev. B 62, 11556 (2000).

[34] R. Robles and S. N. Khanna, Phys. Rev. B 82, 085428 (2010).

[35] A. B. Shick, F. Máca, and A. I. Lichtenstein, Phys. Rev. B 79, 172409 (2009).

[36] M. A. Barral, P. Roura-Bas, A. M. Llois, and A. A. Aligia, Phys. Rev. B 82, 125438 (2010).

[37] O. Šipr, S. Bornemann, J. Minár, and H. Ebert, Phys. Rev. B 82, 174414 (2010).

[38] S. K. Panda, I. Di Marco, O. Grånäs, O. Eriksson, and J. Fransson, Phys. Rev. B 93, 140101 (2016).

[39] M. Ternes, New J. Phys. 17, 063016 (2015). 